\title{
Susceptibility of Chinese Hamsters (Cricetulus griseus) to the Infection of Babesia microti
}

\author{
Kazunori IKE ${ }^{1)}$, Takashi MURAKAMI'), Tetsuya KOMATSU'1), Yuko UCHIDA ${ }^{1)}$ and Soichi IMAI ${ }^{1)}$ \\ ${ }^{1)}$ Department of Veterinary Parasitology, Nippon Veterinary and Animal Science University, Musashino, Tokyo 180-8602, Japan
}

(Received 14 May 2004/Accepted 16 November 2004)

\begin{abstract}
Chinese hamsters were examined for the susceptibility to the infection with Babesia microti based on the hematological parameters during the course of infection. A marked decease in the RBC count, $\mathrm{Ht}$ value, $\mathrm{Hb}$ concentration, and an increase in WBC count due to the development of neutrophils or monocytes were recognized with the progress of parasitemia. Remarkable clinical findings were anemia and persistent infection with a low level of parasite burden in the chronic and convalescent stages. From these findings, it was concluded that Chinese hamsters were susceptible to infection with B. microti and would be useful for infection examination with the parasite.
\end{abstract}

KEY WORDS: Babesia microti, Chinese hamster, susceptibility.

J. Vet. Med. Sci. 67(3): 333-336, 2005

Babesiosis is caused by the protozoan blood parasites of the genus Babesia which causes pyrexia, hemolytic anemia, defluxion of hemoglobinuria. These parasites multiply in the erythrocytes of host animal and cause destruction of blood cells as a cardinal symptom of babesiosis [15]. The disease is widespread in both domestic and wild animals throughout the world and is an important infectious disease in veterinary clinics [6]. Babesiosis caused by $B$. microti arouses interest also as an emerging zoonosis in humans [12]. The cases have been reported especially in North America, where human babesiosis is caused mainly by $B$. microti and occasionally by a newly recognized species of parasite called WAI piroplasma [3, 20]. However, in Europe, the human babesiosis is rather infrequent but more lethal and mostly caused by $B$. divergens [19]. In addition, very recently a new pathogenic $B$. microti-like species was detected from dogs and humans in Germany and Japan, respectively $[18,28]$. In field conditions, small rodents are important reservoir hosts of the parasite [24], which are generally cause parasitemia in enzootic regions and chronic infection persists in less than $0.5 \%$ of the erythrocytes $[1$, 13]. Thus, experimental rodent models are necessary for the immunological investigation on the interactions between $B$. microti parasite and its susceptible hosts in chronic infection with low parasitemia. Syrian hamsters (Mesocricetus aura$t u s$ ) have high susceptibility to $B$. microti infection and are used to isolate and maintain the parasite in laboratories [4, $16,21]$, but the hamsters are not suitable rodent reservoir hosts to control the infection with the parasite, because they show spontaneous remission with low parasitemia [8]. Several species of mice are more commonly used for experimental infections with the parasite, but the mouse-adapted parasites are essential for experimental infections [22]. The rodents are as small as mice and susceptible to $B$. microti as well as Syrian hamsters, so they will be useful for infection experiments with the parasite. The Chinese hamster (Cricetulus griseus) was first used as a laboratory animal in 1919
[10]. The benefits such as small size, polyestrous cycle, short gestation period, and a small number of chromosome encouraged the use of the Chinese hamster in biomedical researches [10]. The Chinese hamster was shown to be susceptible to a number of parasites, such as Spirometra erinacei [11], Necator americanus [5], Schistosoma mansoni [2], S. japonicum [14], Trichinella spiralis [25], T. pseudospiralis [25] and Acanthamoeba keratitis [26]. In this paper, we studied the susceptibility and utility of Chinese hamsters to the infection with $B$. microti because of easy breeding and comfortable handling.

Female Chinese hamsters, 10-week-old, originated from a closed, conventionally outbred, laboratory colony, were maintained in the laboratory animal facility at Nippon Veterinary and Animal Science University. Animal experimentation was carried out according to the Laboratory Animal Control Guidelines at Nippon Veterinary and Animal Science University. To determine the infective dose of $B$. microti to hamster, 2 animals each were infected with doses from $1 \times 10^{5}$ to $1 \times 10^{9}$ parasitized erythrocytes $(\mathrm{PE})$ prepared by serial ten-fold dilution of these originated from Syrian hamsters infected with the parasite, and were clinically observed until 8 weeks post infection. In the following examination, 10 Chinese hamsters were inoculated intraperitoneally with $1 \times 10^{7} \mathrm{PE} /$ head from the infected Syrian hamsters (infection group), while other 10 hamsters were not inoculated (control group). Five-week-old female Syrian hamsters were purchased from Saitama Experimental Animals Supply Co., Ltd. (Saitama, Japan) and used for passage and experimental infections with the parasite. $B$. microti strain AJ used was isolated from a human patient at Harvard University, U.S.A., and maintained by blood passages with Syrian hamsters in Nippon Veterinay and Animal Science University.

Parasitemia (PE rate) in the peripheral circulation was monitored throughout the observation period by making a thin blood smear from a drop of blood from the hamsters at 
Table 1. Parasitemia of Chinese hamsters post inoculation with several dose of Babesia microti

\begin{tabular}{cccccc}
\hline \multirow{2}{*}{$\begin{array}{c}\text { Dose / } \\
\text { head }\end{array}$} & $\begin{array}{c}\text { No. of } \\
\text { hamsters }\end{array}$ & \multicolumn{4}{c}{ Average parasitemia post infection (\%) } \\
\cline { 3 - 6 } & 2 weeks & 4 weeks & 6 weeks & 8 weeks \\
\hline $1 \times 10^{5}$ & 2 & ND* & ND & ND & ND \\
$1 \times 10^{6}$ & 2 & ND & 0.8 & ND & ND \\
$1 \times 10^{7}$ & 2 & 19.0 & 17.4 & 4.9 & 1.9 \\
$1 \times 10^{8}$ & 2 & 26.3 & 10.1 & 2.2 & 0.8 \\
$1 \times 10^{9}$ & 2 & $\mathrm{~d}^{* *}$ & & & \\
\hline
\end{tabular}

* ND: Not detected, ** d: Died within $24 \mathrm{hr}$ after infection.

the tip of tail or the infraorbital vein, and the rate was determined by counting at least 1,000 erythrocytes per smear stained with Diff-Quik (International Reagents Corporation, Hyogo, Japan).

Hematological examinations were done as follows: blood was collected from Chinese hamsters at the tail or infraorbital vein. Erythrocyte (RBC) and leukocyte (WBC) counts, hemoglobin concentration $(\mathrm{Hb})$, and packed cell volume (PCV) were determined with the blood of hamsters by using the mouse mode of Celltac $\alpha$ (Nihon Kohden, Tokyo, Japan). Neutrophils, eosinocytes, basophilocytes, lymphocytes and monocytes were counted by the microscopic observation of blood smears.

Fifteen weeks post infection (PI), all infected Chinese hamsters were subcutaneously given with Dexamethasone (Sigma, U.S.A.) at the dose of $0.2 \mathrm{mg} / \mathrm{kg} 2$ times at $48 \mathrm{hr}$ intervals.

Average parasitemia of Chinese hamsters infected at several infective doses of $B$. microti is shown in Table 1 . The hamsters infected with $1 \times 10^{7}$ and $1 \times 10^{8} \mathrm{PE}$ showed high PE rates, 19.0 and $26.3 \%$, and 17.4 and $10.1 \%$ at 2 and 4 weeks post infection, respectively. However, the hamsters infected with $1 \times 10^{5}$ and $1 \times 10^{6} \mathrm{PE}$ could hardly showed parasitemia until 8 weeks PI, and those with $1 \times 10^{9} \mathrm{PE}$ died within $24 \mathrm{hr}$ PI.

In the following examination, all Chinese hamsters were readily infected with $B$. microti after inoculated with the infected Syrian hamster's blood. The patterns of parasitemia PI in the Chinese hamsters are shown in Fig. 1(A). $B$. microti merozoites first appeared at 2 weeks PI in the peripheral blood. The average value of parasitemia progressively increased and peaked synchronously in all the hamsters at 3 weeks PI (25.3\%). Parasitemia values subsequently declined to 5 and $1 \%$ at 5 and 8 weeks PI, respectively. At the end of experiment at 15 weeks PI, the parasites were scarcely detected in the peripheral blood $(<$ $1 \%$ ). From the parasitemia patterns in Chinese hamsters infected with $B$. microti, the course of infection was divided into 3 stages, the latent ( $0-2$ weeks PI), acute (2-5 weeks $\mathrm{PI})$, and chronic and/or convalescent stages ( 5 weeks $\mathrm{PI}<$ ). More than 8 weeks PI in the chronic stage, the parasites still detected in the peripheral blood. After administering of dexamethasone at 15 weeks PI, parasitemia was temporarily increased at 16 weeks PI, but then quickly decreased at 17 or 18 weeks PI. These findings suggest that the Chinese ham- sters infected with $B$. microti are of latent infection in the chronic stage.

In the domestic animals infected with Babesia species, parasitemia observed in blood smears is usable as an index to show affected degree of the animal [7]. PE rate of an affected animal in natural infections shows sudden increase followed by a short latent period, and after that, the rate decreases to the lower level in the chronic or convalescent stage with asymptomatic or latent infection [23]. A similar pattern in the change of the PE rate was also reported in the experiments on the splenectomised calves infected with $B$. ovata and B. bigemina $[7,27]$ and on the dogs infected with $B$. gibsoni [16]. Compared with these findings, Chinese hamsters infected with $B$. microti are assumed useful for the choronic and convalescent stage model of babesiosis.

Results of RBC counts, $\mathrm{PCV}$, and $\mathrm{Hb}$ values are shown in Fig. 1(B), 1(C), and 1(D), respectively. RBC counts, PCV and $\mathrm{Hb}$ values fell by about $50 \%$ of the preinfection level at 3 weeks PI. After that, these parameters were gradually recovered, but these values did not exceed the those of the control group by 15 weeks PI. From these results, it is considered that erythrocytes are destructed in parallel with the multiplication of parasite in the RBCs. These erythroid changes were also provided in the experimental infection of splenectomy cows with $B$. ovata and B. bigemina [7, 27]. Although canine babesiosis with $B$. gibsoni develops progressive anemia in spite of low parasitemia, it shows the same type of pathogenesis or macrocytic anemia as bovine babesiosis [9].

Changes of WBC, neutrophil and monocyte counts in the Chinese hamsters infected with B. microti are shown in Fig. $1(\mathrm{E}), 1(\mathrm{~F})$, and $1(\mathrm{G})$, respectively. WBC counts increased with a rise of parasitemia. This increase was due to the development of mature neutrophils and monocytes. It is known that WBC counts fall slightly at first, but they recover two- to three-fold levels over those of preinfection due to the increase in lymphocytes [17]. There are several reports on the hematological examination of WBC parameters in babesiosis of calves and dogs [7, 17, 27], but their results were not consistent. Such difference in WBC parameters may be influenced by the difference in pathogenesis of various Babesia species.

In general, Syrian hamsters are used to isolate and maintain $B$. microti in laboratories due to their high susceptibility to the parasite $[4,16,21]$. However, the hamsters are not 

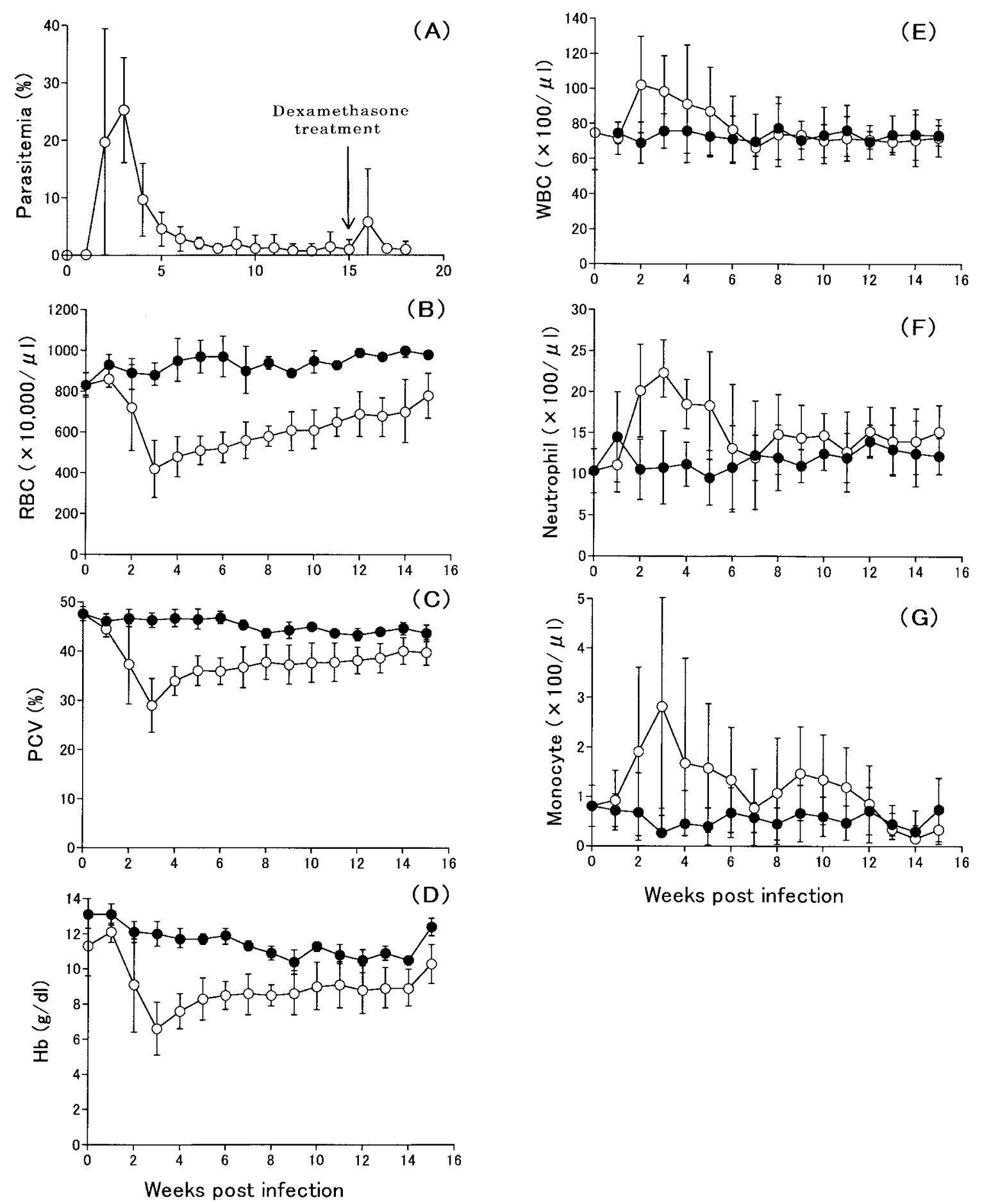

Weeks post infection

Fig. 1. Parasitemia (A), RBC (total erythrocyte count) (B), PCV (packed cell volume) (C), Hb (hemoglobin concentration) (D), WBC (total leukocyte count) (E), neutrophil count (F), and monocyte count (G) estimation in the blood of Chinese hamsters infected with Babesia microti. $\bigcirc$ : Infection group, : Control group.

suitable to control infection as reservoir rodent hosts showing spontaneous remission with low parasitemia of less than $0.5 \%$ infected erythrocytes in the chronic and convalescent stages [8]. Mice are susceptible for mouse-adapted $B$. microti strains, but not so for the primary infection with the strains maintained in Syrian hamsters [22]. In this study, we 
confirmed that Chinese hamsters were susceptible to the infection with $B$. microti and would be useful for the infective examination with the patasite.

\section{REFERENCES}

1. Bajer, A., Pawelezyk, A., Behnke, J. M., Gilbert, F. S. and Sinski, E. 2001. Parasitology 122: 43-54.

2. Carbtree, J. E. and Wilson, R. A. 1984. Parasitology 89: 5969.

3. Dammin, G. J., Spielman, A., Benach, J. L. and Piesman, J. 1981. Hum. Pathol. 12: 398-400.

4. Etkind, P., Piesman, J., Ruebush, J. K. II, Spielman, A. and Juranek, D. D. 1980. J. Parasitol. 66: 107-110.

5. Evans A. C., Daly, T. J. and Markus, M. B. 1991. J. Helminthol. 65: 67-72.

6. Friendhoff, K. T. 1988. pp. 23-52. In: Babesiosis of Demestic Animals and Man (Ristic, M. ed.), CRC Press Inc., Florida.

7. Fujinaga, T. 1981. Jpn. J. Vet. Sci. 43: 803-813.

8. Gleason, N. N., Healy, G. R., Western, K. A., Benson, G. D. and Schultz, M. G. 1970. J. Parasitol. 56: 1256-1257.

9. Groves, M. G. and Dennis, G. L. 1972. Exp. Parasitol. 31: 153-159.

10. Hankenson, F. C. and van Hoosier Jr., G. L. 2002. pp. 167202. In: Laboratory Animal Medicine 2nd ed. (Fox, J. G., Anderson, L. C., Loew, F. M. and Quimby, F. W. eds.), Academic Press Inc., New York.

11. Hirai, K., Shiwaku, K., Tsuboi, T., Torii, M., Nishida, H. and Yamane, Y. 1983. Z. Parasitenkd. 69: 489-499.

12. Homer, M. J., Aguilar-Delfini, Telford III, S. R., Krause, P. J. and Persing, D. H. 2000. Clin. Microbiol. Rev. 13: 451-469.

13. Karbowiak, G. and Sinski, E. 1996. Acta Parasitol. 41: 50-51.

14. Kutsumi, H., Inaoka, T. and Ohnishi, K. 1988. Hokkaido Igaku Zasshi 63: 80-84 (In Japanese).
15. Kuttler, K. L. 1988. pp. 1-22. In: Babesiosis of Demestic Animals and Man (Ristic, M. ed.), CRC Press Inc., Florida.

16. Lewis, G. E. and Huxsoll, D. L. 1976. pp. 1330-1336. In: Current Veterinary Therapy VI (Kirk, R. W. ed.), W.B., Saunders Co., Philadelphia.

17. Mahoney, D. F. 1977. pp. 1-52. In: Parasitic Protozoa, vol. IV, (Kreier, J. P. ed.), Academic Press Inc., New York.

18. Matsui, T., Inoue, R., Kajimoto, K., Tamekane, A., Katayama, Y., Shimoyama, M., Chihara, K., Saito-Ito, A. and Tsuji, M. 2000. Jpn. J. Clin. Hematol. 41: 628-634 (in Japanese with English summary).

19. Musa, N. B. and Philips, R. S. 1991. Parasitology 103: 165 170.

20. Persing, D. H., Herwaldt, B. L., Glaser, C., Lane, R. S., Thomford, J. W., Mathiesen, D., Krause, P. J., Phillip, D. F. and Conrad, P. A. 1995. New Engl. J. Med. 332: 298-303.

21. Piesman, J. and Spielman, A. 1982. Exp. Parasitol. 53: 242 248.

22. Ruebush, M. J. and Hanson, W. L. 1979. J. Parasitol. 65: 430433.

23. Schalm, O. W., Jain, N. C. and Carroll, E. L. 1975. pp. 405470. In: Veterinary Hematology, 3rd ed. (Schalm, O. W., Jain, N. C. and Carroll, E. J. eds.), Lea and Febiger, Philadelphia.

24. Spielman, A., Etkind, P., Piesman, J., Ruebush, T. K. II, Juranek, D. D. and Jacobes, M. S. 1981. Am. J. Trop. Med. Hyg. 30: 560-565.

25. Stewart, G. L. and Larsen, E. 1989. J. Parasitol. 75: 10061007.

26. van Klink, F., Alizadeh, H. He, Y., Mellon, J. A., Silvany, R. E., McCulley, J. P. and Niederkorn, J. Y. 1993. Invest. Ophthalmol. Vis. Sci. 34: 1937-1944.

27. Wright, I. G. 1973. Res. Vet. Sci. 14: 29-34.

28. Zahler, M., Rinder, H., Schein, E. and Gothe, R. 2000. Vet. Parasitol. 89: 241-248. 